\title{
Understanding the bi-directional relationship between Psoriasis and Mental Health
}

\author{
Serat Rana \\ BSc Psychology (Honours), Christ (Deemed to be University), 2020 Batch \\ Corresponding author: Serat Rana \\ Email - seratrana6@gmail.com
}

\begin{abstract}
Introduction
Psoriasis is a chronic autoimmune disease that causes the rapid build-up of skin cells. This build-up of cells causes scaling on the skin's surface. Inflammation and redness around the scales is fairly common. Typical psoriatic scales are whitish-silver and develop in thick, red patches. They may develop anywhere on the body, including the hands, feet, neck, scalp and face. Less common types of psoriasis affect the nails, the mouth, and the area around genitals. Psoriasis may begin at any age, but most diagnoses occur in adulthood. The average age of onset is between 15 to 35 years old. According to the World Health Organization (WHO), some studies estimate that about 75 percent of psoriasis cases are diagnosed before age 46 . Several other conditions that psoriasis can be associated with are type 2 diabetes, inflammatory bowel disease, heart disease, psoriatic arthritis, anxiety, depression etc. There are different types of psoriasis such as the plaque, guttate, pustular, inverse and erythrodermic psoriasis. The American Academy of Dermatology (AAD) estimates that about 80 percent of people with the condition have plaque psoriasis.

One of the causes of this condition is genetics. Some people inherit genes that make them more likely to develop psoriasis. However, the percentage of people who have psoriasis and a genetic predisposition is small. Approximately 2 to 3 percent of people with the gene develop the condition, according to the National Psoriasis Foundation (NPF) The other is the immune system, autoimmune conditions are the result of the body attacking itself. In the case of psoriasis, white blood cells known as T cells mistakenly attack the skin cells. In a typical body, white blood cells are deployed to attack and destroy invading bacteria and fight infections. This mistaken attack causes the skin cell production process to go into overdrive. The sped-up skin cell production causes new skin cells to develop too quickly. They are pushed to the skin's surface, where they pile up. This results in the plaques that are most commonly associated with psoriasis. The attacks on the skin cells also cause red, inflamed areas of skin to develop. Many external factors lead to the development or aggravation of this condition. Among known environmental triggers of psoriasis, there are drugs, infections, physical trauma, smoking, alcohol, and stress [1].

Psoriasis can be diagnosed through biopsies and physical examinations. Topical treatments and light therapy are recommended to control the symptoms of psoriasis. Since there is no cure, managing the effects is crucial and can be improved by a healthy diet and lifestyle. People with psoriasis are more likely to experience depression and self-esteem issues. Psoriasis impairment to psychological quality of life is comparable to cancer, myocardial infarction, and depression [2]. One may feel less confident when new spots appear. A new hypothesis linking depression and psoriasis through chronic inflammation offers insights that should help to understand and treat these diseases [3]. Psychological issues such as depression, anxiety, body image, self-esteem as a result of this condition in studies have been highlighted. Since stress is a well-established trigger for the condition, a bidirectional relationship between psoriasis and mental health can be discerned. Understanding the psycho-social aspect of living with this condition can improve the management of symptoms, and even help in avoiding the consequent adverse effects.
\end{abstract}

\section{Biological Perspectives on Psoriasis and Depression}


The connection between psoriasis and depression has been discovered substantively. It is usually believed that psoriasis may lead to the development of depression. However, studies show that psoriasis can be caused by depression owing to immunological and neurochemical phenomena. According to a study, high levels of proinflammatory cytokines (TNF- $\alpha$ and IL-6) are found in patients suffering from depression, usually in the absence of any inflammatory disease. In contrast, chronic inflammation can cause various mood disorders, including depression [4]. There is also a strong genetic influence on psoriasis and depression. Polymorphism of the serotonin receptors due to genetic differences leads to the development of depressive and psoriatic symptoms. Research on skin diseases, inflammation and depression suggest a strong biological basis and overlap. Genetic evidence, inflammatory overlap, low vitamin D3, and melatonin levels in both psoriasis and depression set up a connection between these two diseases [4]. Using a Hospital Anxiety and Depression Scale (HADS) in Spain, a dermatology study explored the impact of skin diseases on anxiety and depression. It was observed that the more serious the dermatological lesion, the higher the score on depression [5]. Another study done to measure the BDI (Beck's Depression Inventory) score of psoriasis patients with a control group, depicted that the scores were much higher in the former group. Whereas interviews with psoriasis patients suggest that stress and depression trigger psoriasis flare- ups, indicating a reversal mechanism.

\section{Impact of Psoriasis on Body Image and Self Esteem}

One's perception about themselves, especially about their body, is known as body image. Body image is a central component of one's self image and self-esteem and contributes substantially to the mental health of an individual. Extensive research has explored body image and self-esteem across various settings. It is only natural that skin disorders such as psoriasis one's evaluation of their bodies and their intimacy with others. One qualitative paper has reported (briefly) on sexual aspects of the wider patient experience of living with severe psoriasis and found some participants' sexual lives to have been affected, mainly by the physical effects of psoriatic lesions but also by feelings of unattractiveness and disgust regarding lesions [6]. A study done to understand dyadic adjustment and family coping in patients with psoriasis and their partners found that their negative body image affected their identity as sexual beings and their intimacy levels with their partners. Difficulties in body image, shifts in the social network and decreased sexual intimacy as a result of psoriasis can be triggers for chronic stress, threatening patients' and partners' well-being as well as relationships with others [7]. A study of 1981 included participants who had psoriasis were asked to draw human figures and the results were gauged on the basis of extent of sexual focus, nudity and omission of parts. Conclusions of the study revealed that higher body image concerns are related to the extent of psoriatic severity in individuals. The substantial differences in the omission of parts in mild and severe psoriasis suggest greater anxiety levels in the latter. Some gender differences were also observed as psoriatic women depicted less clothes on their drawings compared to the psoriatic male with the same severity of symptoms. An analysis of interviews of people with skin disorders including psoriasis around the themes of bullying and teasing as a means of social exclusion elaborated on the diseases' effect on one's self image. For those who had suffered teasing or bullying, this was causally linked in some respondents' accounts with emotional or psychological sequelae, especially self-consciousness and effects on self-image and self-esteem [8]. An interesting concept of "double jeopardy" was put forth by a study in which participants with psoriasis were asked to visualise the extent of discriminations in specific domains and otherwise. Both males and females felt that the people with psoriasis are more disadvantaged compared to others. However, in the social domain and in a work-related social setting, women showed a smaller personal-group discrimination discrepancy than did men with psoriasis because of higher ratings for personal discrimination [9]. Indicating that women feel more perturbed in social settings owing to the fact they are women living with psoriasis. To understand the willingness for social direct and indirect contact with people with dermatological conditions revealed that the participants were more inhibited when maintaining indirect contact with a person with acne and psoriasis as compared to the control group with no skin condition. This can be further elucidated by the research done by Schaller and Park that explains the concept of "behavioural immune system" which leads us to detect "potential pathogens". A visually different appearance might cue pathogen fear and a response indicative of reactive avoidance, such as being less willing to share items with the person. These processes operate in the absence of objective evidence of potential infectiousness [10]. It is also noted that internalised 
stigma is strengthened in cases of psoriasis that are more visually prominent to others, making those individuals less keen to interact socially and leading to intrapersonal aversion. People with psoriasis, the stigmatization experience seems to be better captured by their fear and avoidance of disgust reactions of others. Indications toward a social threat related bias were found in psoriasis [11]. Therefore, it can be inferred by the findings of the above-mentioned studies, that psoriasis has a strong negative influence on one's body image, self-esteem and even social relationships.

\section{Stress and Psychological Morbidity and Psoriasis}

Stress is a common factor in everyone's life. However, chronic distress can cause hindrance to one's daily life and overall well-being. Psoriasis can be a stress- responsive disease for some, therefore there is a strong link between the two. Cortisol acts as an inhibitor of inflammation as a response to emotional and physical stress, and the functioning of cortisol is shown to be dysfunctional in people with psoriasis. Although the cortisol response is a good clue to what might be going on, it's not very specific, cortisol levels fluctuate for many reasons. So researchers are investigating how stress affects some cells and molecules thought to play a specific role in plaque formation in psoriasis. A study executed the Trier test, a strategy for inducing stress in social settings, on people with psoriasis. Some reported flare-ups before the task and those who did had lower cortisol levels compared to others. The effect of stress on psoriasis symptoms is complicated by the fact that psoriasis itself causes stress. Stress responders also tend to have more psoriasis on highly visible areas of the body, such as the face and hands [12]. Somatisation is also discussed in cases of self-induced rashes due to PTSD and other such distresses which trigger or aggravate one's skin problems. The autonomic instability and hyper-arousal in PTSD may cause a flare-up of a stress-reactive dermatosis [13] such as psoriasis, eczema or atopic dermatitis [14]. There is increasing appreciation among scientists for this psycho dermatological association and aspect of diseases. A study done to understand the effects of psychological stress in psoriasis patients found that illness perception was largely influenced by worrying and alexithymia (a condition that causes difficulty in understanding and describing emotions), more so than clinical history and other variables. Patients with psoriasis show marked differences in the processing of emotionally valenced stimuli compared with controls, suggesting a hypervigilance for threat leading to attempted avoidance of such threat [15]. An overlap between existence of alexithymia and higher anxiety levels is suggested in the results of the study. Research done on Chinese patients with psoriasis extrapolated that patients reported considerably higher scores for depression and anxiety. The patients were categorised as stress reactors and non-stress reactors and it was seen that positive stress reactors were more susceptible to mood disorders. Positive stress reactors and higher PASI (Psoriasis Area and Severity Index) scores had a negative effect on the presence of moderate-to-severe depression or anxiety symptoms while longer duration of psoriasis and late onset age played a protective role [16]. A study of a selected multi-ethnic Malaysian population with psoriasis discussed some interesting notions such as certain personality traits and the effect it may have on stress levels. Such as Type A personalities put more pressure on themselves to succeed that can lead to higher levels of stress in workplaces and even generally. An alternative explanation for this unique finding could be that individuals with tertiary levels of education with psoriasis were more likely to develop stress from having psoriasis and individual appearance holds a greater value among those with higher levels of education, with those with lower levels of education less likely to be stressed by the presence of a dermatological condition [17]. This understanding was reinforced by a Mongolian study in which an educational level was a deciding factor for anxiety levels. Social avoidance in different age groups and gender differences were present, noting that women had higher scores of depression. Gupta and Gupta's study [13] shows that younger psoriatic patients $>45$ report lower quality of life. It is also observed that psychological reactions and reporting of the same was displayed more by women as compared to their male counterparts. When clinical variables and psoriasis were explored in a study, $43 \%$ felt psoriasis burdened their everyday life. Patients with psoriasis may be vulnerable with respect to psychosocial functioning and well-being as a result of physical symptoms and disfigurement [6]. When emotional regulation and psychopathology in psoriatic patients was studied, an association between emotional regulation problems and the condition was apparent. Emotional regulation difficulty was significantly correlated with psychopathological symptoms. Patients with a higher number of psychopathological symptoms, particularly anxiety, depression, and obsessive-compulsive symptomatology, experienced greater difficulty in emotional 
regulation [18]. A higher chance of comorbidity with psychiatric disorders in psoriatic patients is depicted in many studies leading to an increased risk of suicidal ideation compared to patients with other dermatological problems. Noteworthy, a positive history of past suicide attempts was found only among patients with psoriasis [19].

\section{Coping and Therapeutic Interventions and Psoriasis}

Coping is defined by Lazarus and Folkman as "Constantly changing cognitive and behavioural efforts to manage, reduce or tolerate external and/or internal demands that are appraised as taxing or exceeding the resources of the person". Coping is a process and therefore depends on the situational context in which it occurs [20]. It is often used as a mechanism to deal with and adapt to distress and illnesses. A psoriasis patient's cognitive appraisal of their condition, definition of adaptive tasks and selection of coping skills are influenced by the person themselves, by aspects of the transition or crisis, and by their environment [21]. There are several types of coping strategies that one adopts. One study distinguished between emotive and problem focused coping strategy. Emotive coping is one in which one engages in acts such as wishful thinking to deal with their problems whereas problem focused has an information seeking approach. It was observed that psoriatic patients that employed emotive coping strategies had a low mental health and quality of life as compared to the ones who used normalizing coping strategies. It was suggested that beliefs of being in control of the illness and seeking social support are effective coping strategies in this arena. A study done to understand the psychological health of psoriatic included the disadvantages of negative coping. Our results showed that negative coping had the greatest influence on the mental health of psoriasis patients [22]. Positive active coping such as humor, planning were employed less by individuals with psoriasis as compared to the control group in a study. Although venting emotions was chosen frequently as an effective coping strategy. Stigmatization research in skin disorders points to an avoidance-based approach in psoriatic patients as a response to social threats such as disgusted faces. This finding is confirmed by another study that also adds that avoidance coping leads to higher anxiety levels. Distorting and ignoring are part of avoidance coping which is only beneficial for a short duration of time. It is also known to contribute to the existence of psychiatric disorders such as anxiety and depression. Whereas approach coping is observed as an effective way to combat stress and in ensuring a sound mental health. There is an emerging need for clinical interventions and patient education to aid illnesses such as psoriasis and its adverse effects. One intervention that could be worth applying to such a patient group is the acceptance and commitment therapy (ACT), which aims to alter patients' attitudes toward and the coping with their (chronic) disease [23].

A study that explored family coping and dyadic adjustment of psoriatic patients suggested that it is essential to focus on psychosocial interventions. Future studies should address the impact of psoriasis on the couple's developmental phase, patients' and partners' illness representations, caregiver burden and how all these variables are related to dyadic adjustment and quality of life in both patients and partners [7]. This notion was supported by a study that focused on emotional regulation in psoriatic participants, urged to formulate interventions that include emotional regulation processes for patients who are struggling to cope. Adversarial growth is an interesting and less researched concept in disease management. The idea is to focus on the positives of the struggles one goes through in a difficult journey, and being optimistic to facilitate growth and adaptive skills. Results suggest that adversarial growth is related to greater psychological mindedness, awareness of the reality of disease course, and being younger at onset of the condition [15]. Studies also reveal that alexithymia or difficulties in processing emotions has a negative influence on growth, indicating a need to adopt an attitude of acceptance and reorganisation. Essentially, the experience of stress aids in the neural pruning and reconfiguration that is essential to adversarial growth [15]. A research plan including patient education and climate therapy was found to be effective in dealing with physical and psychological symptoms in psoriatic patients. The content of this treatment included sun treatment, physical workouts such as hiking, gym, etc, in combination with healthy lifestyle changes and interactions with people with similar health struggles. However the maintenance of benefits after going back home post the treatment was not successful. Working along the lines of Pavlov's classical conditioning, a Medical Resonance Therapy - Music was conducted in a hospital to assess its effectiveness on psoriatic patients. Combination of regular hospital treatment and few hours of MRT-Music per day, led to reduction of degree of sickness by $65 \%$ in the patients, adding to the arena of modern stress management.Stress reduction 
interventions could be particularly useful for psoriasis stress responders [24]. A study done on Cognitive Psoriasis Management Programme(PSMP) improved self-perceptions about the illness after a 6 month follow up in participants. Cognitive Behavioral Therapy (CBT) that works on the basis of identifying and altering maladaptive thoughts and beliefs is highly recommended by most researchers. We have conducted a controlled trial to ascertain the utility of the CBT program. At the end of a six-week intervention, we found that patients who received CBT in addition to their regular topical or systemic therapy had less severe psoriasis than the patients who didn't have the CBT [25]. MBCT (Mindfulness Behavioral Cognitive Therapy) builds on this mechanism in tandem with Mindfulness Stress Reduction Therapy are popular intervention choices for health management and especially for psoriasis. The aim of these therapies is to diminish sources of stress by instilling a sense of acceptance and awareness of challenging scenarios presented in their illness. A study in which MBCT with a clinically modified Buddhist model was employed on people with psoriasis for 8 weeks in group participation. The findings showed that the approach oriented skills improved and so did their mental and physical health. Self compassion was observed as a cross situational benefit in MBCT participants, which proved to be pivotal in combating worries and anxiety linked with the illness. A common pattern emerged in the interview data, all interview participants reported that they experienced reductions in either rumination or worry [26]. However, like most interventions the long term effectiveness is dubious.

\section{REFERENCES}

1. Villanova F, Flutter B, Tosi I, Grys K, Sreeneebus H, Perera GK, Chapman A, Smith CH, Di Meglio P, Nestle FO. Characterization of innate lymphoid cells in human skin and blood demonstrates increase of NKp44+ ILC3 in psoriasis. J Investig Dermatol 2014;134(4):984-91.

2. Rendon A, Schäkel K. Psoriasis pathogenesis and treatment. Int J Molecular Sci 2019;20(6):1475.

3. Dauden E, Blasco AJ, Bonanad C, Botella R, Carrascosa JM, González-Parra E, Jodar E, Joven B, Lazaro P, Olveira A, Quintero J. Position statement for the management of comorbidities in psoriasis. J Eur Acad Dermatol Venereol 2018;32(12):2058-73.

4. Sahi FM, Masood A, Danawar NA, Mekaiel A, Malik BH. Association Between Psoriasis and Depression: A Traditional Review. Cureus 2020;12(8).

5. Moya S, Aragones L. An Epidemiological Study to assess the psychological impact on the quality of life and personality traits of patients with psoriasis in the Calatayud Health Area. J Am Acad Dermatol 2005;52(3):1906.

6. Wahl A, Hanestad BR, Wiklund I, Moum T. Coping and quality of life in patients with psoriasis. Qual Life Res 1999;8(5):427-33.

7. Pereira MG, Brito L, Smith T. Dyadic adjustment, family coping, body image, quality of life and psychological morbidity in patients with psoriasis and their partners. Int J Behav Med 2012;19(3):260-9.

8. Magin PJ, Pond CD, Smith WT, Watson AB, Goode SM. A cross-sectional study of psychological morbidity in patients with acne, psoriasis and atopic dermatitis in specialist dermatology and general practices. $\mathrm{J}$ Eur Acad Dermatol Venereol 2008;22(12):1435-44.

9. Perrott SB, Murray AH, Lowe J, Mathieson CM. The psychosocial impact of psoriasis: physical severity, quality of life, and stigmatization. Physiol Behav 2000;70(5):567-71.

10. Farber EM, Nall L. Psoriasis in the tropics: epidemiologic, genetic, clinical, and therapeutic aspects. Dermatol Clin 1994;12(4):805-16.

11. Van Beugen S, Van Middendorp H, Ferwerda M, Smit JV, Zeeuwen-Franssen ME, Kroft EB, de Jong EM, Donders AR, van de Kerkhof PC, Evers AW. Predictors of perceived stigmatization in patients with psoriasis. Br J Dermatol 2017;176(3):687-94.

12. DeWeerdt S. Psychodermatology: an emotional response. Nature 2012;492(7429):S62-3.

13. Gupta MA, Gupta AK, Watteel GN. Early onset (< 40 years age) psoriasis is comorbid with greater psychopathology than late onset psoriasis: a study of 137 patients. Acta Dermatovenereol 1996;76(6):464-6.

14. Gupta MA, Gupta AK. Depression and suicidal ideation in dermatology patients with acne, alopecia areata, atopic dermatitis and psoriasis. Br J Dermatol 1998;139(5):846-50.

15. Fortune DG, Richards HL, Griffiths CE. Psychologic factors in psoriasis: consequences, mechanisms, and interventions. Dermatol Clin 2005;23(4):681-94.

16. Tian S, Krueger JG, Li K, Jabbari A, Brodmerkel C, Lowes MA, Suárez-Fariñas M. Meta-analysis derived (MAD) transcriptome of psoriasis defines the "core" pathogenesis of disease. PloS One 2012;7(9):e44274. 
17. Kwan Z, Bong YB, Tan LL, Lim SX, Yong AS, Ch'ng CC, Tan MP, Ismail R. Determinants of quality of life and psychological status in adults with psoriasis. Arch Dermatol Res 2018;310(5):443-51.

18. Almeida V, Taveira S, Teixeira M, Almeida I, Rocha J, Teixeira A. Emotion regulation in patients with psoriasis: Correlates of disability, clinical dimensions, and psychopathology symptoms. Int J Behav Med 2017;24(4):563-70.

19. Pompili M, Innamorati M, Forte A, Erbuto D, Lamis DA, Narcisi A, Rea C, Orsini D, D'Arino A, Arcese A, Bellini S. Psychiatric comorbidity and suicidal ideation in psoriasis, melanoma and allergic disorders. Int J Psychiatr Clin Pract 2017;21(3):209-14.

20. Folkman S, Lazarus RS. Stress, appraisal, and coping. New York: Springer Publishing Company; 1984.

21. Creswell C, Chalder T. Defensive coping styles in chronic fatigue syndrome. J Psychosom Res 2001;51(4):60710.

22. Zhang Q, Han J, Zhang Y, Li C, Chen P, Zhang J, Zeng K. Study on the psychological health and related risk factors in 245 patients with psoriasis in Inner Mongolia. Psychol Health Med 2019;24(7):769-80.

23. Schut C, Muhl S, Reinisch K, Claßen A, Jäger R, Gieler U, Kupfer J. Agreeableness and self-consciousness as predictors of induced scratching and itch in patients with psoriasis. Int J Behav Med 2015;22(6):726-34.

24. Fordham B, Griffiths CE, Bundy C. A pilot study examining mindfulness-based cognitive therapy in psoriasis. Psychol Health Med 2015;20(1):121-7.

25. Griffiths CE, Richards HL. Psychological influences in psoriasis. Clin Experiment Dermatol 2001;26(4):33842.

26. Maddock A, Hevey D, D'Alton P, Kirby B. Examining Individual Differences in Wellbeing, Anxiety and Depression in Psoriasis Using a Clinically Modified Buddhist Psychological Model. J Clin Psychol Med Settings 2020;27(4):842-58.

$$
\begin{gathered}
\text { Acknowledgements - Nil } \\
\text { Conflict of Interest - Nil } \\
\text { Funding - Nil }
\end{gathered}
$$

